\title{
Characterization of Cysteine Coated Magnetite Nanoparticles as MRI Contrast Agent
}

\author{
Reza Ahmadi ${ }^{1,2, *}$, Ning $\mathrm{Gu}^{3}$, Hamid Reza Madaah Hosseini ${ }^{1}$
}

(Received 16 September 2012; accepted 20 September 2012; published online 25 September 2012.)

\begin{abstract}
In this work, a kind of stabilized ferrofluid based on magnetite nanoparticles (mean core and its coating size about 21.9 and $1.6 \mathrm{~nm}$, respectively) was synthesized via coprecipitation method. Cysteine was used as surfactant due to its proper conjunction to the surface of magnetite nanoparticles. Coating density and synthesized ferrofluids were characterized by using transmission electron microscope, thermogravimetry analysis, dynamic light scattering and fourier transform infrared spectroscopy techniques. Magnetic resonance imaging studies show that the synthesized ferrofluid can be used as a potential contrast enhancement agent especially for imaging lymphatic system.
\end{abstract}

Keywords: Magnetite; Co-precipitation; Cysteine; MRI

Citation: Reza Ahmadi, Ning Gu and Hamid Reza Madaah Hosseini, "Characterization of Cysteine Coated Magnetite Nanoparticles as MRI Contrast Agent", Nano-Micro Lett. 4 (3), 180-183 (2012). http://dx.doi. org/10.3786/nml.v4i3.p180-183

\section{Introduction}

Ferrofluids containing magnetic nanoparticles have potential applications in drug delivery, cancer therapy, magnetic resonance imaging (MRI) contrast enhancement agent, etc [1-5]. One of the fundamental preconditions for mentioned medical applications is high stability of synthesized ferrofluids with time and environmental variations. Among various parameters affecting this stability, surfactant layer density plays an important role.

Formation of a high density coating will prevent agglomeration or sedimentation of solid phase. Besides, the nature and composition of surfactant is an important factor. Cysteine with three functional groups have a certain binding affinity to metal atoms especially $\mathrm{Fe}$ atoms, so can be effectively conjugated into magnetite nanoparticles' surface [3].

Ultrasonic-assisted co-precipitation method provides conditions for narrow size distribution and uniform thickness of coating around the nanoparticles. This is due to uniform nature of ultrasonicating irradiation and cavity bubble formation in various areas of reaction container.

It seems that the most accurate and reliable method of measuring coating thickness and density is direct observation of coating layer via electron microscopy, which is based on mass contrast as the main mechanism. Since amorphous organic surfactants can not reveal diffraction contrast mechanisms similar to crystalline phases, detection and recognition of these layers from crystalline magnetic core is rather a technical process that requires good understanding of transmission electron microscope (TEM) principles. One of the main subjects in this case is that surfactant layers usually can be seen only at low voltages in the order of $60-100 \mathrm{kV}$, the range at which resolution is not suitable for imaging of nanometric phases.

Rutherford equation [6] presents probability or fraction of scattering phenomenon $\left(\frac{\partial \sigma}{\partial \Omega}\right)$ from phase of $\mathrm{Z}$

\footnotetext{
${ }^{1}$ Department of Materials Science and Engineering, Sharif University of Technology, P.O. Box 11155-9466, Tehran, Iran

${ }^{2}$ Research Center for Molecular and Cellular Imaging, Tehran University of Medical Sciences, Tehran, Iran

${ }^{3}$ Jiangsu Key Laboratory of Biomaterials and Devices, Nanjing, China

*Corresponding author. E-mail: reahmady@yahoo.com
} 
molecular weight at accelerating voltage of $\mathrm{V}$,

$$
\frac{\partial \sigma}{\partial \Omega} \alpha \frac{Z^{4}}{V}
$$

According to Equation (1), TEM study of organic compounds of Cysteine type containing low atomic weight elements such as Hydrogen and Carbon should be performed at low voltages. Besides, TEM study at intermediate should be performed with low illumination, i.e. low dose of electron density due to beam damage. In many publications [7-9] surfactant layers are not visible in presented TEM images due to the mentioned reasons.

In order to detect surfactant layer directly and measure its thickness, synthesized nanopaticles were studied at two various levels of accelerating voltage in this work. Comparison of measured thickness by thermogravimetry analysis (TGA) and dynamic light scattering (DLS) results provides possibility of evaluation of coating density via some simple calculations. This approach can be used in similar cases. Finally, synthesized ferrofluid with suitable hydrodynamic size was injected intravenously in rat to evaluate its functionality as magnetic resonance imaging (MRI) contrast agent.

\section{Experimental}

All chemical reagents in this study were of analytical grade and used as received without further pu- rification. The detailed process is described elsewhere [3]. Briefly speaking, co-precipitation procedure performed using $\mathrm{FeCl}_{3} \cdot 6 \mathrm{H}_{2} \mathrm{O}$ and $\mathrm{FeCl}_{2} \cdot 4 \mathrm{H}_{2} \mathrm{O}$ as Fe resources, $\mathrm{NaOH}$ as reducing agent and Cysteine as surfactant. $\mathrm{N}_{2}$ blowing was employed through three neck balloon. Optimizing experimental conditions such as temperature, $\mathrm{pH}$, molar ratio of reactants, ultrasonicating frequency and power, magnetite Cysteine capped nanoparticles were synthesized. Black precipitates were removed from liquid phase, washed, centrifuged, rewashed with distilled water and hexane and finally dried and prepared for characterization experiments. Some suspension samples were prepared for DLS tests, whereas TGA and fourier transform infrared spectroscopy (FT-IR) samples were dried powders. TEM samples were prepared via conventional method in which a suspension drop is trickled on the Carbon coated grid surface and studied after droplet drying.

ZEISS EM-10C and high resolution Philips CM200 TEMs were used to determine the average particle and coating sizes, and morphology of the powders at accelerating voltage of 80 and $200 \mathrm{kV}$, respectively. FT-IR was performed using a Nicolet Magna 500 instrument. Malvern 4.2 DLS instrument was employed for hydrodynamic diameter measurement. TGA was performed using TGA Q50 instrument in order to investigate specimen weight changes. MRI performed with a $1.5 \mathrm{~T}$ (GE medical system) by using a knee coil for transmission and reception of the signal.
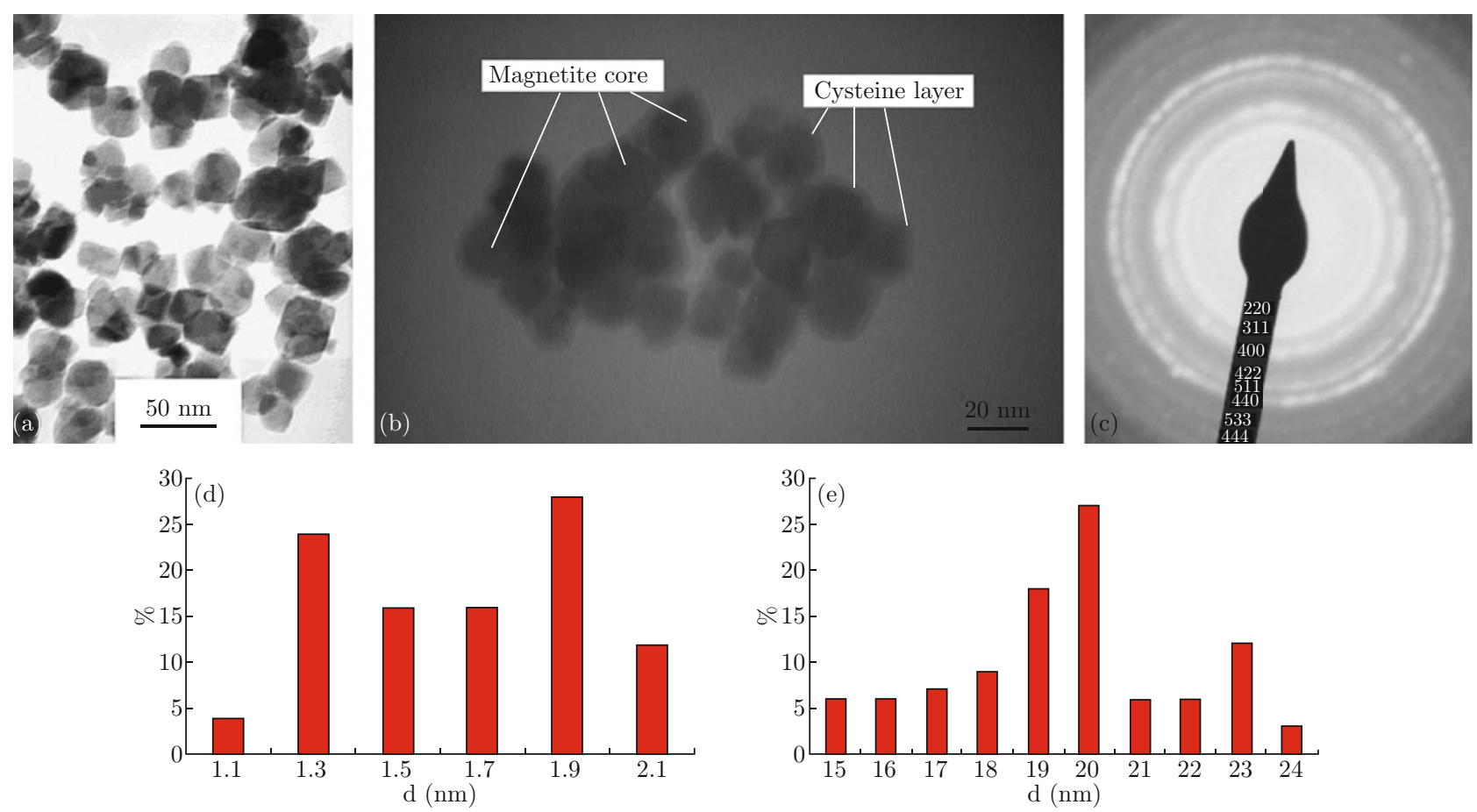

Fig. 1 TEM image of synthesized nanopartcles at (a) $200 \mathrm{kV}$ and (b) $80 \mathrm{kV}$. (c) Related diffraction pattern showing spinel structure of magnetite. (d) Coating thickness histogram and (e) particle size histogram obtained from Fig. 2(b). 


\section{Results and discussion}

TEM images of the synthesized ferrofluid at accelerating voltage of 200 and $80 \mathrm{kV}$ and its diffraction pattern are shown in Figs. 1(a), 1(b) and 1(c), respectively. Spinel structure of magnetite can be upheld from Fig. 1(c) comparing crystallographic planes distances of synthesized nanoparticles with those of pure $\mathrm{Fe}_{3} \mathrm{O}_{4}$. Figure 1(a) shows that particles shape lies mainly between spherical and cubic pattern. No surfactant layer can be recognized in this high voltage. Attempts made for this reorganization were not efficacious. On the other hand, a dim ring can be seen in lower voltage, $80 \mathrm{kV}$ (Fig. 1(b)). This uniform ring surrounded all particles and has a mean thickness of about $1.6 \mathrm{~nm}$ (Fig. 1(d)). Figure 1(e) shows the particle size histogram obtained from Fig. 1(b) with mean particle size of $21.9 \mathrm{~nm}$. Hydrodynamic size histogram of prepared sample obtained by DLS measurement is shown in Fig. 2. According to this histogram mean hydrodynamic size equals to $36.4 \mathrm{~nm}$, which is appropriate for imaging or drug delivery to lymph nodes [10].

XRD pattern of the synthesized sample is presented in Fig. 3. Six characteristic peaks for $\mathrm{Fe}_{3} \mathrm{O}_{4}$ nanoparticles marked by their indices (220), (311), (400), (422), (511) and (440), were recognized for this sample. These peaks are well matched with the magnetite characteristic peaks (JCPDS card no. 19-0629) confirming the inverse spinel structure of the particles in coincidence with TEM results.

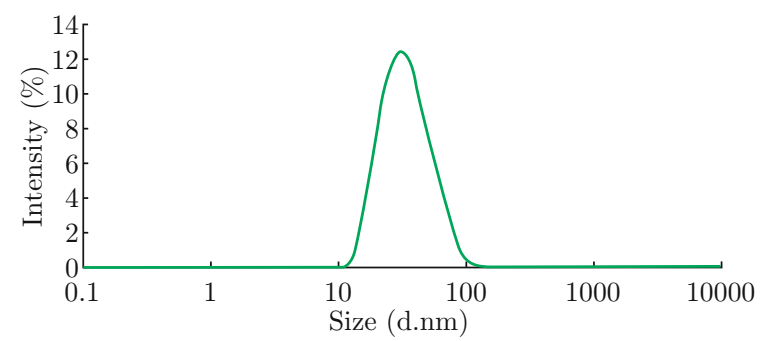

Fig. 2 Size histogram of the synthesized nanoparticles obtained from DLS measurement. Mean hydrodynamic diameter of this sample is $36.4 \mathrm{~nm}$.

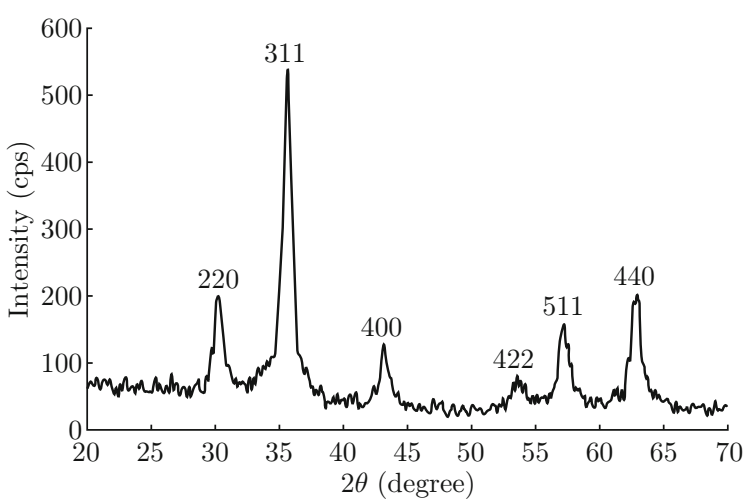

Fig. 3 XRD pattern of the synthesized cysteine coated magnetite nanoparticles.
Figure 4 shows FT-IR results of $\mathrm{Fe}_{3} \mathrm{O}_{4}$ in the range of $4000-400 \mathrm{~cm}^{-1}$. The adsorption band at $592 \mathrm{~cm}^{-1}$ is the stretching mode of $\mathrm{Fe}-\mathrm{O}$ in $\mathrm{Fe}_{3} \mathrm{O}_{4}$ [11]. The other bands among $1650-1100 \mathrm{~cm}^{-1}$ are assigned to the stretching of ethyl and carbonyl groups. The characteristic absorptions of alkyl stretching modes between 2800 and $3200 \mathrm{~cm}^{-1}$ are recognized as vibration of functioned groups of Cystein on the surface of $\mathrm{Fe}_{3} \mathrm{O}_{4}$ nanoparticles.

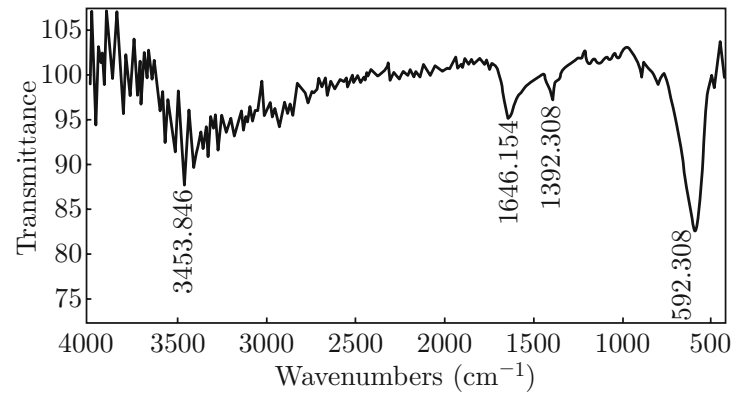

Fig. 4 FT-IR spectrum of nanoparticles.

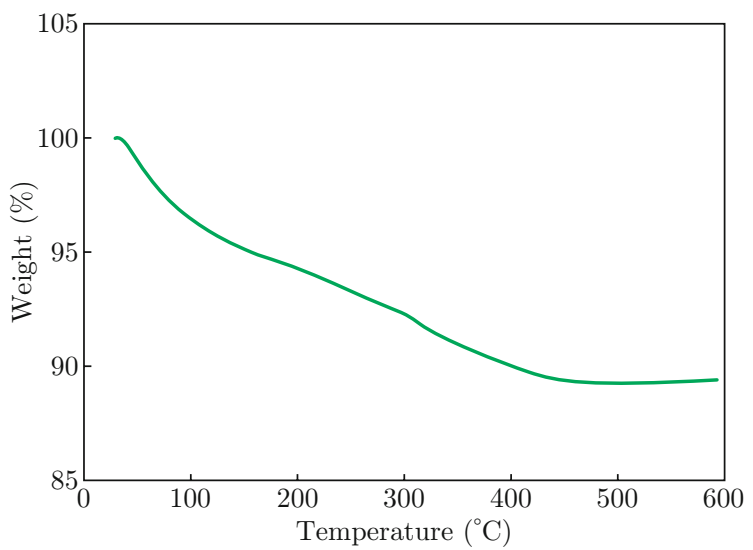

Fig. 5 TGA diagram of synthesized nanoparticles.

Figure 5 shows that weight loss percent due to decomposition of probably organic phase is about $9.73 \%$. As the sample was vacuum dried at $100^{\circ} \mathrm{C}$ before TGA, this weight loss is due to Cyctein decomposition. Some simplifying assumptions can be accomplished to calculate coating density. First, magnetic core is pure magnetite. This assumption is in good agreement with diffraction pattern of the sample (Fig. 1(c)) and XRD and FT-IR spectra (Figs. 3 and 4), which shows only Fe-O band belongs to Oxygen and Iron ions covalent band in $\mathrm{Fe}_{3} \mathrm{O}_{4}$ chemical structure [10]. Thus, core density can be assumed equal to $5.15 \mathrm{~g} / \mathrm{cm}^{3}$ [12]. Second, magnetic core has a nearly spherical shape. Third, average coating thickness surrounding all particles is about $1.6 \mathrm{~nm}$. TEM image (Fig. 1(b)) confirms this assumption. Forth, mean magnetite core size is about 21.9 nm (TEM Figs. 1(b) and 1(e)). Using these assumptions, coating density is simply calculated to be 1.09 $\mathrm{g} / \mathrm{cm}^{3}$. Comparing with solid L-Cysteine density of $1.67 \mathrm{~g} / \mathrm{cm}^{3}[12]$, this means that a rather high density coating is formed that satisfies fundamental conditions 
of formation of a stable ferrofluid. This is in good agreement with this fact that synthesized ferrofluid in this work were stable for about 2 months (Fig. 6).

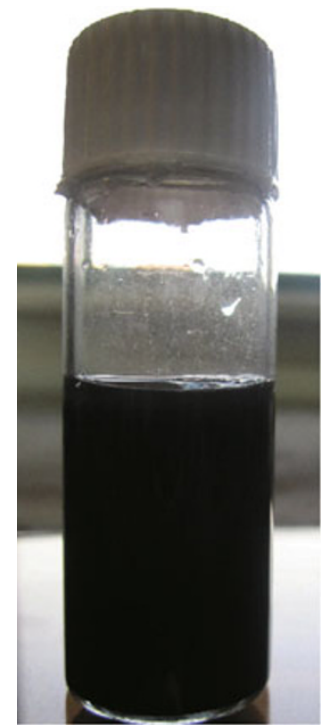

Fig. 6 The stabilized ferrofluid containing cysteine coated magnetite nanoparticles after 2 months (concentration: 2 $\mathrm{mgFe} / \mathrm{ml})$.

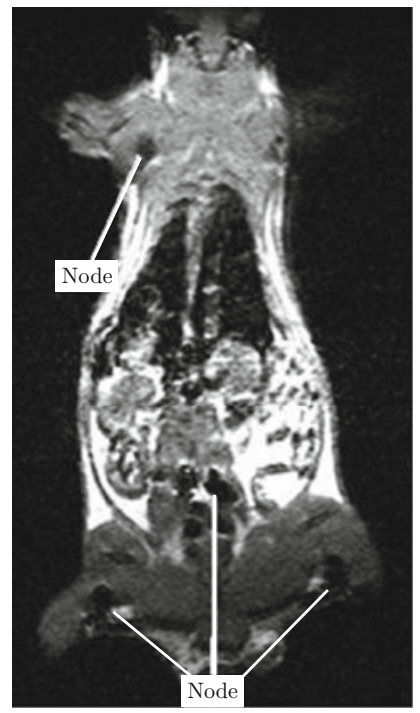

Fig. 7 MRI results of rat $24 \mathrm{~h}$ after IV injection.

For MRI study, rat was anaesthetized by pentobarbital sodium at the dose of $40 \mathrm{mg} / \mathrm{kg}$ body weight. Synthesized nanoparticles stabilized through the aqueous ferrofluid were administered into rat's bloodstream via lateral tail vein at a dose of $2.5 \mathrm{mg}(\mathrm{Fe}) / \mathrm{kg}$ body weight. MRI scan was performed $24 \mathrm{~h}$ after administration. As is explained by theory [10], larger and aggregated particles are mainly accumulated in tissues such as liver and spleen, however, smaller ones (20-40 $\mathrm{nm})$ are phogositosed by macrophages of lymphatic system (Fig. 7). Enhancement of these tissues through substantial shortening of $\mathrm{T} 2$ relaxation times leads to hypo signal intensity of related tissues on MRI. Figure 5 demonstrates signal enhancement in the area of lymph nodes 24 hours after injection of synthesized ferrofluid.

\section{Conclusion}

A stable ferrofluid with respective mean magnetic core size and coating thickness of about 21.9 and $1.6 \mathrm{~nm}$ was synthesized using ultrasonic-assisted coprecipitation method. Synthesized ferrofluid had mean hydrodynamic diameter of $36.4 \mathrm{~nm}$. Coating density was calculated as $1.09 \mathrm{~g} / \mathrm{cm}^{3}$. This shows that a high density coating is formed on the nanoparticles surface. In animal study, good MRI contrast enhancement was seen for lymph nodes after IV injection of synthesized ferrofluid.

\section{References}

[1] J. Zhang, S. Rana, R. S. Srivastava and R. D. K. Misra, Acta Biomat. 40, 4 (2008).

[2] B. Cyrille, R. W. Michael, B. Volga, L. Jingquan and P. D. Thomas, NPG Asia Mater. 2, 23 (2010). http: // dx.doi.org/10.1038/asiamat. 2010.6

[3] R. Ahmadi, M. Malek, H. R. Madaah Hosseini, M. A. Shokrgozar, M. A. Oghabian, A. Masoudi, N. Gu and Y. Zhang, Mat. Chem. Phys. 131, 170 (2011). http: // dx.doi.org/10.1016/j.matchemphys. 2011.04.083

[4] H. B. Ki et al., ACS Nano 6, 5266 (2012). http://dx. doi.org/10.1021/nn301046w

[5] E. Karaoğlu, H. Kavas, A. Baykal, M. S. Toprak and H. Sözeri, Nano-Micro Lett. 3, 79 (2011). http://dx. doi.org/10.3786/nml.v3i2.p79-85

[6] D. B. Williams and C. B. Carter, Transmission Electron Microscopy: A Textbook for Materials Science, 1st edn., Plenum Press, New York, 1996.

[7] K. M. Spiers, J. S. Forsythe, K. Suzuki and J. D. Cashion, J. Magn. Magn. Mater. 311, 97 (2007). http:// dx.doi.org/10.1016/j.jmmm.2006.10.1191

[8] D. H. Kim, S. H. Lee, K. H. Im, K. N. Kim, K. M. Kim, I. B. Shim, M. H. Lee and Y.-K. Lee, Curr. Appl. Phys. 6, 242 (2006). http://dx.doi.org/10.1016/j. cap. 2006.01.048

[9] X. Wang, H. Gu and Z. Yang, J. Magn. Magn. Mater. 293, 334 (2005). http://dx.doi.org/10.1016/ j.jmmm . 2005.02.028

[10] L. LaConte, N. Nitin and G. Bao, Materials Today 8, 32 (2005). http://dx.doi.org/10.1016/ S1369-7021 (05) 00893-X

[11] R. Y. Hong, T.T. Pan and H.Z. Li, J. Magn. Magn. Mater. 303, 60 (2006). http://dx.doi.org/10.1016/ j.jmmm . 2005.10.230

[12] R. C. Weast, CRC Handbook of Chemistry and Physics, 70th edn., CRC Press, Boca Raton, 1990. 